Federal Reserve Bank of Minneapolis

Research Department Staff Report 279

September 2000

\title{
Growth Cycles and Market Crashes
}

\author{
Michele Boldrin* \\ Federal Reserve Bank of Minneapolis \\ and University of Minnesota
}

David K. Levine*

University of California, Los Angeles

\begin{abstract}
Market booms are often followed by dramatic falls. To explain this requires an asymmetry in the underlying shocks. A straightforward model of technological progress generates asymmetries that are also the source of growth cycles. Assuming a representative consumer, we show that the stock market generally rises, punctuated by occasional dramatic falls. With high risk aversion, bad news causes dramatic increases in prices. Bad news does not correspond to a contraction of existing production possibilities, but to a slowdown in their rate of expansion. This economy provides a model of endogenous growth cycles in which recoveries and recessions are dictated by the adoption of innovations.

*We are grateful to Fundacion Marc Rich, DGICYT PB97-0091, NSF grant SBR-9617899 and the UCLA Academic Senate for support. The paper benefited greatly from comments by two anonymous referees and from conversations with Boyan Jovanovic, Jose Wynne and Bill Zame. Jose Wynne carried out the simulations. The views expressed herein are those of the authors and not necessarily those of the Federal Reserve Bank of Minneapolis or the Federal Reserve System.
\end{abstract}




\section{Introduction}

Whenever there is a dramatic fall in the stock market, public discussion centers on the idea that investor psychology or irrationality is the root cause. Closely associated with this view is the idea that stock market crashes (and the booms that precede them) are a bad thing. Even within professional circles, the fact that the stock market rises relatively smoothly, but falls abruptly is used as evidence for this point of view. Indeed, to explain the asymmetry in rises and falls, a fundamentalist view of the stock market requires an asymmetry either in the underlying technology shocks that drive fundamentals or in the information-processing mechanisms that characterize the market. The first source of asymmetry has been recently studied by Zeira [18]; the second source of asymmetry has been studied, for example, in a recent paper by Lee [13]. In this paper we consider the first case, that is, an asymmetry in the underlying process of technological improvement.

On the face of it, such an asymmetry seems implausible. Our goal is to argue, on the contrary, that a very natural model of technological progress under uncertainty leads to exactly the type of asymmetry required to explain gradual rises in the value of the capital stock, punctuated by sharp declines, provided that traders are not terribly risk averse. Indeed our contention is that this same model can explain both asymmetric growth cycles with long recoveries and short and sharp recessions, and asymmetric movements of stock market prices. This is accomplished without the need for the unpalatable assumption of technological regress. The evidence presented in Crafts [5], David [6], Hornstein and Krusell [11], among others, suggests that large recessions may be triggered by technological innovations and the adoption of new machinery.

Greenwood and Jovanovic [8] and [9] make a related point. They argue that when a technology plays out, the stock market declines. This may occur because incumbent firms resist the introduction of the new technology, or it may be because of the need to train labor as in Greenwood and Yorukoglu [10]. After a period of time, the new technology is successfully introduced and the stock market rebounds. They focus on the IT revolution as a case study of the replacement of one technology (main frame computers) by another (PCs). Unlike in our argument, however, the market does not recover gradually, but rather suddenly, as the new technology comes on line all at once. 
The idea that a switch-over between the old and new technology leads to an economic slowdown can be found in many other places; Atkeson and Kehoe [2] is one example. Another is Zeira [18], which is closely connected in spirit and basic intuition to this paper. Like us, Zeira stresses the role played by the arrival of information on stock market movements and the intrinsic asymmetry between the "good news," corresponding to a continuation of the growth process, and the "bad news," corresponding to a sudden halt of the growth process. Zeira, however, concentrates on the learning process induced by the arrival of new information, while we focus on the endogenous growth and technological innovation side of the problem.

We should also note that we study a one input (capital) model of production, as for example, in Lucas [14] or Mehra and Prescott [16]. As a result, our model does not address the controversial issue of correlations between labor inputs and technology shocks, discussed, for example, in Gali [7].

Our basic model is that of an economy growing due to improving technology introduced in Boldrin and Levine [4]. In this model, activity analysis is used to model the existing state of knowledge as a set of currently available activities. This set of available activities grows over time due to technological progress. In general, to make use of new activities, it is necessary to introduce new kinds of capital. In this setting, it is natural to distinguish between research and development-the process of introducing new technologies and the process of improving old ones. We model this by assuming that there are many types of capital, each one corresponding to a different underlying technology. In addition, each technology (type of capital) is available in many generations. The most rapid form of technological improvement is to upgrade existing capital to a newer generation. Treating research as "extensive" and development as "intensive," Jovanovic and Rob [12] have derived exactly this result in a fairly general micro-model of endogenous technological process. This distinction is also consistent with evidence from the applied industrial organization literature. This literature suggests that new technologies require a long time to be adopted on a large scale and go through prolonged periods of productivity enhancement before improvement opportunities die out. See, for example, Mansfield [15]. 
In our model each type of capital (technology) is limited in how many generations of improvement can be sustained: no matter how much effort we put into it, an improved bicycle never turns into an automobile. Nevertheless the stage of improvement at which a capital stock is played out and can be improved no further is not known in advance. When a technology is played out, however, it is possible to introduce a new type of capital, but there is necessarily a time delay before the new capital can compete on equal terms with the old type of capital.

We study an activity analysis economy with constant returns to scale and zero profits. At each moment of time, capital is a fixed factor and is priced according to the expected discounted value of future rents, which are in the form of consumption produced directly or indirectly from that capital. In equilibrium, this price, computed from marginal rates of transformation, is equal to the price computed from marginal rates of substitution, such as those used in Lucas [14].

In this economy the basic technology shock is the discovery that an existing type of capital is played out. What impact does this have on the value of the existing capital stock? The playing out of an existing type of capital does not reduce the currently available activities or capital in any way, but it does make future production possibilities less attractive than they would have been if the technology had not played out. The impact this has on the market value of the capital stock depends (in our case of CES preferences) on the coefficient of relative risk aversion. If the coefficient of relative risk aversion is positive, then the value of capital increases; if it is negative, it decreases. This is due to the combined impact of the bad news on future interest rates and on future consumption flows. In the highly risk averse case, interest rates drop sufficiently that even though the value of future consumption to which current capital is a claim goes down, the present value actually goes up. This, maybe surprising, feature of asset prices is not special to our model. It applies to all consumption-based asset pricing models which adopt a time-separable, CES utility function to value uncertain consumption streams. For example, in simulations with the Mehra-Prescott [16] model, we find that with the coefficient of relative risk aversion greater than one, feeding observed consumption shocks into the model results in stock price movements of an empirically relevant magnitude, but the wrong sign. This underlying mechanism also means that when 
consumption growth shocks are positively correlated, the risky asset is a good hedge against risk, so that with high risk aversion the risk premium is actually negative. This was first pointed out in Boldrin, Christiano and Fisher [3].

Given our specification for preferences, the case of low risk aversion is therefore the empirically interesting one, because it corresponds to the stock market dropping on bad news. We explore in some detail the time path of capital, consumption and prices in the case in which technology shocks are relatively infrequent. Ordinarily there is no bad news, and the value of capital gradually increases over time due to the overall growth in consumption. This is the period of stock market booms. However, following a bad shock, the value of capital falls abruptly. At this time, a new type of capital is introduced and used to produce additional generations of the new type of capital, until eventually they are sufficiently productive to be used in the production of consumption. At this time, the old type of capital is retired, and the economy resumes its previous growth.

Of particular interest is the transition. Although this period corresponds to one of technological innovation in the sense that a new kind of capital is being produced for the first time, it is also a recession. In particular during the transition following the negative shock, consumption and GNP may rise or decline, but they rise less rapidly than in good times. During the recession, net investment stalls and a replacement process from old to new machines takes place. The stock of capital grows at the same slow rate that consumption does. During this recession, the stock market gradually recovers from its initial fall.

We also examine the quantitative implications of the theory, showing that in a realistic range of parameter values, it is possible to generate falls in the stock market on the order of 10-20\%, together with plausible business cycles.

Key to this point of view is the idea that neither the stock market boom, the stock market crash, nor the recession that follows the crash are bad. Here they are part of a first best solution to the problem of maximizing the present value of utility from consumption. Of course, the negative shock is a bad thing in the sense that it would be better if it were possible for technology to continue improving. However, there is nothing in the working of the stock market or in the behavior of consumption after the crash that could be improved upon. 
At the heart of our results is the underlying idea that technology shocks are properly thought of as asymmetric. Take, for example, "Moore's Law," the "law" that says that the speed of microprocessors doubles every 18 months. From basic physical principles, we are confident that this improvement will not continue forever. However, it is difficult to predict with certainty when it will come to a stop. The discovery that Moore's law no longer holds, when it happens, may occur quite abruptly. Consider, on the other hand, the discovery of a new technology, however, abrupt: there is necessarily a delay while the new capital is built, the new technology deployed, and the practical experience (development) needed to use it effectively takes place. It is this basic asymmetry that we capture here. This asymmetry is especially important when we consider technological change in a broader perspective. For, at the aggregate level, technology is not merely the knowledge of how to build things. It is also the institutions and arrangements for trade, and capital includes the knowledge of how best to make use of those institutions. So, for example, in a developing country, changes in legal arrangements such as trade policy or improvements in financial markets can be viewed as the introduction of a new technology. However, it takes some time for producers and traders to learn how best to make use of these new institutions. In our model this corresponds to upgrading the current type of capital. However, the benefits, for example, of trade liberalization, are not unlimited; in our model, it is the discovery that benefits of liberalization have been exhausted that triggers a recession and a fall in the stock market.

The rest of the paper is organized as follows. The next section looks at the behavior of the S\&P 500 index over the twentieth century and concludes that the empirical evidence rejects the hypothesis that stock market movements are driven by a symmetric Markov process. Section 3 introduces our formal model and shows its equilibria are efficient. Section 4 characterizes the dynamic properties of such equilibria, showing, in particular, the differential impact on the value of the capital stock of bad and good news. We present both qualitative and quantitative evidence of this fact by simulating the growth cycles generated by the model. Section 5 concludes. 


\section{Is There An Asymmetry Between Rises and Falls?}

The first issue is whether there is evidence of the conventional wisdom of asymmetry in rises and falls of the stock market. The purpose of the simple data analysis we are going to present next is not to test our model, although detailed testing would be valuable in future work, but rather to show that asymmetry is potentially a real puzzle that needs to be explained. We should point out that our explanation of asymmetric shocks or responses to shocks is not the only one-see, for example, Zeira [18] and Lee [13] for interesting models of how informational dynamics can lead to stock market overshooting and corresponding asymmetries.

In the Appendix, we reproduce from Shiller [17] the real Standard and Poor's Index annually from 1889-1984. We also calculate the logarithmic differences from one year to the next as a deviation from the mean. The mean logarithmic growth rate during the period was $1.0 \%$.

The "common wisdom" we wish to verify is that increases are smaller and more persistent than decreases. In the table below we compute the length of runs of deviations above (+) and below (-) the mean growth.

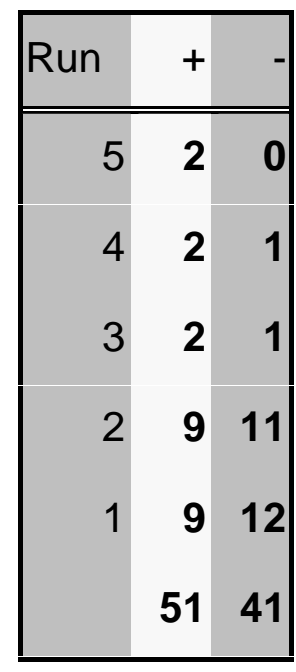

This provides some preliminary evidence of asymmetry: runs of positive deviations are longer than those with negative deviations, and there are more positive than negative deviations. The fact that there are more positive than negative deviations is also reflected in the fact that positive deviations are on average smaller than negative 
deviations: the average positive deviation is $12.3 \%$, while the average negative deviation is $-16.0 \%$. This difference of $3.7 \%$ is reasonably large in economic terms.

If shocks to growth are symmetric around the mean and i.i.d., how likely is it that these differences are due to sampling error? A simple test is to fit a two state Markov model in which the probability of the next sign depends on the previous sign. The maximum likelihood estimates are derived by computing the frequency of + separately, conditional on the previous sign. If the previous sign was + , the probability of another + is $53 \%$; if the previous sign was -, the probability of $a+$ is $61 \%$. We can also compute standard errors for each of these coefficients, subtract $50 \%$ and normalize: conditional on + the normalized value is $41 \%$; conditional on - the normalized value is $141 \%$. The probability that the first value is $41 \%$ or more (using the normal approximation to the binomial) is $34 \%$; the probability that the second value is $141 \%$ or more is $8 \%$. Since the two values are independent of one another under the i.i.d. assumption, the probability that both events occur is just the product of the two probabilities: $2.7 \%$. So if the data are truly symmetric and i.i.d. there is only a $2.7 \%$ chance of the observed discrepancy.

\section{The Model}

\subsection{The State Space}

We use a standard infinite horizon event tree model of time and uncertainty. Dates are denoted by $t=0,1,2, \ldots$. At each date $t$ there are possible a countable number of states $\eta_{t} \in I$. A state history $s=\left(\eta_{0}, \eta_{1}, \ldots, \eta_{t}\right)$ is a finite history of states. The (countable) set of all state histories is denoted by $S$. For a given state history $s=\left(\eta_{0}, \eta_{1}, \ldots, \eta_{t}\right)$, we let $t(s)=t$ denote the terminal time and $\eta_{t}$ the terminal state. We also order state histories so that $\tilde{s} \geq s$ if $t(\widetilde{s}) \geq t(s)$ and $\tilde{\eta}_{\tau}=\eta_{\tau}$ for $\tau \leq t(s)$. We write $s-1$ for the immediate predecessor of $s$ and $s^{+}$for the set of immediate followers of $s$. We let $\pi_{s}$ denote the probability of state history $s$, so that $\sum_{s: t(s)=t} \pi_{s}=1$.

\subsection{Households}

There is a single representative household, who following the state history $s$ consumes $c_{s} \in \Re_{+}$. This household receives total lifetime utility of $\sum_{s \in S} \delta^{t(s)} u\left(c_{s}\right) \pi_{s}$, where $0 \leq \delta<1$ is a subjective discount factor. Rather more strongly, we will assume that 
the period utility function is smooth, concave, bounded below, and, at least for levels of consumption above a subsistence level $c \geq \underline{c}$, has the CES (or constant relative risk aversion) form $u(c)=-(1 / \theta) c^{-\theta}$, where $\theta+1 \geq 0$ is the coefficient of relative risk aversion. Note that for $\theta \geq 0$ the CES function is not bounded below, which is why we require this functional form only for levels of consumption above subsistence. As we are interested in the theory of growth, not the theory of subsistence, the behavior of $u$ for small quantities of consumption is not terribly interesting. In particular, in the equilibria we consider, consumption will be uniformly bounded away from zero, and we will assume that this bound exceeds the subsistence level. This means that we can assume that utility is bounded below, which is technically useful, while nevertheless having the convenience of the CES functional form.

\subsection{Production Possibilities}

There are a countable number of types of capital $\omega=0,1, \ldots$ Each type of capital is potentially available in a countable number of generations $i=\ldots,-1,0,1, \ldots$. We denote by $k^{\omega i}$ the amount of type $\omega$ capital of generation $i$. We refer to a type/generation pair as a kind of capital. Production takes place through linear activities, using capital as input. We begin by describing all potential activities, although not all of these activities are available at any moment of time, and indeed, some activities may never be available.

Each activity takes as input one unit of capital of a specific kind and produces a single output in the following period. Type $\omega$ capital of generation $i$ may be used as the input into 4 distinct activities, each of which is characterized by the output obtained:

1) $\gamma^{i}$ units of the consumption characteristic, $\gamma>1$

2) $\beta>1$ units of the same kind of capital: type $\omega$ capital of generation $i$

3) $\rho: \beta>\rho>1$ units of the next generation of capital: type $\omega$ capital of generation $i+1$

4) 1 unit of type $\omega+1$ capital of generation $i-L, L>0$.

We assume that $\gamma \rho>\beta$, so that more consumption can be obtained by moving to the next generation of capital than by remaining with the current generation. Notice that we do not presume that the increased consumption from higher generations of capital takes place 
through the physical production of increased units of some commodity. The increased amount of characteristic may take place through the production of more advanced commodities which contain more of the characteristic that is actually consumed. However, to keep notation to a minimum, we will not explicitly introduce the distinction between the amount of commodities produced and purchased and the amount of characteristic consumed.

At each moment of time, only a finite number of this countable collection of activities are actually available. The set of available activities is determined by the state, which we take to be a list of those activities that are available. We now describe both the set of possible states and the state transition process that determines $\pi_{s}$, the probabilities of state histories.

A state $\eta \in I$ consists of a latest type of capital $\bar{\omega}(\eta)$, and for each type of capital $\omega \leq \bar{\omega}(\eta)$ a latest generation of capital $\bar{i}(\omega, \eta)$, plus a number $0 \leq m(\eta)$ (this number is used to track the time since the latest type of capital was introduced). The available activities are these: if $\omega \leq \bar{\omega}(\eta), i \leq \bar{i}(\omega, \eta)$ then all four activities making use of $k^{\omega i}$ are available, with the following exceptions:

1) if $\omega<\bar{\omega}(\eta), i=\bar{i}(\omega, \eta)$ then activity 3 producing the next generation of capital is not available

2) if $\omega=\bar{\omega}(\eta), i \leq \bar{i}(\omega, \eta)$ then activity 4 producing the next type of capital is not available.

On the other hand, if $\omega>\bar{\omega}(\eta)$ or $i>\bar{i}(\omega, \eta)$ then no activities making use of $k^{\omega i}$ are possible.

The interpretation of the state is this: except for the latest type of capital, improvements on other types of capital have stalled, and it is no longer possible to produce new generations. New types of capital can be introduced, from the very latest type of capital and its latest generation, only when the opportunity for producing new generations of the latest type of capital has vanished. The latter assumption deserves some comment. It amounts to saying that experimentation with future technologies cannot take place while the current technology is being used and improved. If this were 
not the case, a benevolent planner could take advantage of the constant returns to scale nature of our activities and run the technology $\bar{\omega}+1$ at an infinitesimal scale to acquire valuable information about the generation at which it will play out. This information is valuable because it eliminates the uncertainty about future consumption growth rates. As a result, it also modifies the current market evaluation of the existing stock of capital and makes stock market prices a monotone deterministic process. This assumption is therefore needed to retain a minimum of uncertainty about future technological evolution.

The states follow a Markov process. Fix an initial state $\eta$. We define the next generation of capital as the state $\eta_{g}$ in which $\omega\left(\eta_{g}\right)=\omega(\eta), \bar{i}\left(\omega, \eta_{g}\right)=\bar{i}(\omega, \eta)$ for $\omega<\omega(\eta), \bar{i}\left(\omega(\eta), \eta_{g}\right)=\bar{i}(\omega(\eta), \eta)+1$, and $m\left(\eta_{g}\right)=m(\eta)+1$. We define the next type of capital as the state $\eta_{b}$ in which $\omega\left(\eta_{b}\right)=\omega(\eta)+1, \bar{i}\left(\omega, \eta_{b}\right)=\bar{i}(\omega, \eta)$ for $\omega<\omega(\eta)$, $\bar{i}\left(\omega(\eta), \eta_{b}\right)=\bar{i}(\omega(\eta), \eta)+1, \bar{i}\left(\omega\left(\eta_{b}\right), \eta_{b}\right)=\bar{i}(\omega(\eta), \eta)-L-1$, and $m\left(\eta_{b}\right)=0$. Starting at $\eta$, only $\eta_{g}, \eta_{b}$ can be reached with positive probability. If $m(\eta) \leq M$ then, with probability $1, \eta_{g}$ is reached. On the other hand, if $m(\eta)>M$ with probability $1-\pi$ the state moves to $\eta_{g}$ and with probability $\pi$ the state moves to $\eta_{b}$. This means that for $M$ periods following a transition from state $\eta$ to state $\eta_{b}$ there is no uncertainty about the ability to build new generations of the new type of capital. Since we view $\pi$ as relatively small, this assumption does have much economic import. However, by carefully choosing $M$ we can greatly simplify computations as we indicate below.

We will frequently refer to that transition from $\eta$ to $\eta_{b}$ as a negative or bad shock to technology. Similarly, we will call $\eta_{b}$, which is characterized by $m\left(\eta_{b}\right)=0$, the negative or bad state. But notice that the technological possibilities at $\eta_{b}$ are a strict superset of those available at $\eta$. All activities that were available at $\eta$ are still available at $\eta_{b}$. In particular, we do not assume that a negative shock simply causes the current capital stock to retrogress or become less productive. Indeed, we may wonder why it would ever be desirable to switch to a new kind of capital, which is, after all, essentially identical to the current kind of capital. The answer is that despite the fact that the new kind of capital is actually inferior to the old kind of capital, development of new generations of the old kind of capital is impossible. Switching to the new kind of capital makes it at least possible to introduce higher generations of capital. 


\subsection{Production Plans}

At any moment of time there are in principle a countable number of different types of capital. Let $k_{s} \in X$ be the vector of different kinds of capital when the state history is $s$. The components of $k_{s}$ are $k_{s}^{\omega i}$. An activity may be thought of as a triple $(x, y, c)$, where $x, y \in X$ are the capital inputs in period $t$ and output in period $t+1$, and $c \in \mathfrak{R}_{+}$is the amount of characteristic output in period $t+1$. Let $A$ be the set of all potential activities, and recall that $\eta$ are those actually available when the state is $\eta$. An activity vector $\lambda_{s}$ is a map $\lambda_{s}: A \rightarrow \Re_{+}$. Feasible activities are given by sets $A_{s} \subseteq A$, with $A_{0} \neq \varnothing$ and for $\tilde{s} \geq s$ that $A_{\tilde{s}} \supseteq A_{s}$. A production plan consists of a map from state histories $S$ to capital vectors $k_{s}$, consumption of characteristics $c_{s}$ (for $t(s)>0$ ) and activity levels $\lambda_{s} \in A_{s}$. We say that the production plan is feasible with respect to the initial condition $k_{0}, \eta_{0}$ if for every state history $s$ with $\pi_{s}>0$

$$
\begin{aligned}
\sum_{a \in \eta_{s-1}} \lambda_{s-1}(a) y(a) & \geq k_{s} \\
\sum_{a \in \eta_{s-1}} \lambda_{s-1}(a) c(a) & \geq c_{s} \\
k_{s-1} & \geq \sum_{a \in \eta_{s-1}} \lambda_{s-1}(a) x(a) .
\end{aligned}
$$

We call an activity viable for the state history $s$ and initial condition $k_{0}$ if there exists a socially feasible production plan such that $\lambda_{s}(a)>0$. We denote the set of viable activities for state history $s$ by $A_{s}\left(k_{0}, \eta_{0}\right)$. We assume that the initial set of activities $A_{0}\left(k_{0}, \eta_{0}\right)$ is non-empty for $k_{0} \neq 0$ and that for $\tilde{s} \geq s, A_{\tilde{s}}\left(k_{0}, \eta_{0}\right) \supseteq A_{s}\left(k_{0}, \eta_{0}\right)$. Note that $A_{s}\left(k_{0}, \eta_{0}\right)$ may be a proper subset of the set of feasible activities in $\eta_{s}$. Similarly, we call a kind of capital viable for the state history $s$ and initial condition $k_{0}, \eta_{0}$ if there exists a socially feasible production plan such that there is a positive amount of it available at $s$. Finally, we say that a production plan solves the social planner problem for initial

conditions $k_{0}, \eta_{0}$ if they solve $\max \sum_{s \in S} \delta^{t(s)} u\left(\sum_{a \in \eta_{s}} \lambda_{s-1}(a) c(a)\right) \pi_{s}$ subject to social feasibility.

\subsection{Prices}

Let $q_{s}$ be the present value price of different kinds of capital when the state history is $s$, and let $p_{s}$ be the corresponding present value price of the characteristic. In a competitive equilibrium, these prices should satisfy two conditions: they should yield 
zero profits and support the preferences. Specifically, we say that prices yield nonnegative profits for the initial condition $k_{0}, \eta_{0}$ if

$$
\sum_{\sigma \in s^{+}}\left[p_{\sigma} c(a)+q_{\sigma} y(a)\right]-q_{s} x(a) \leq 0, \forall a \in A_{s}\left(k_{0}, \eta_{0}\right), \forall s
$$

and if this holds with equality, we say that the activity $a$ yields zero-profits. We say that the prices support the consumption plan $\hat{c}_{s}$ if $\hat{c}_{s}$ is a solution to

$$
\begin{gathered}
\arg \max \sum_{s \in S} \delta^{t(s)} u\left(c_{s}\right) \pi_{s} \text { subject to } \\
\sum_{s \in S} p_{s} c_{s} \leq \sum_{s \in S} p_{s} \hat{c}_{s} .
\end{gathered}
$$

The first order conditions for the consumer are satisfied if

$$
\exists \mu>0 p_{s} \geq \mu \delta^{t(s)} \pi_{s} u^{\prime}\left(c_{s}\right) \text { with equality unless } c_{s}=0 \text {. }
$$

Here $\mu$ is the marginal utility of consumption. Note that this first order condition is valid for all values of $c_{s}>0$, but that for $c_{s}<\underline{c}$ the period utility function $u$ does not have the CES form, as we discussed above. The first order condition is most useful in the case of an interior solution where $c_{s-1}, c_{s}>0$, in which case

$$
\delta \frac{\pi_{s}}{\pi_{s-1}} \frac{u^{\prime}\left(c_{s}\right)}{u^{\prime}\left(c_{s-1}\right)}=\frac{p_{s}}{p_{s-1}} .
$$

Finally, we say that prices and capital $k_{s}$ satisfy the transversality condition if

$$
\lim _{T \rightarrow \infty} \sum_{s \mid t(s)=T} q_{s} k_{s}=0 .
$$

\subsection{Efficiency}

We show how to characterize the solution to the planner's problem by a relatively standard price decentralization scheme, despite the fact that there are infinitely many kinds of capital, and those actually available grow over time.

Proposition 3.1: Suppose that $\hat{\lambda}_{s}, \hat{k}_{s}, \hat{c}_{s}$ are socially feasible for the initial condition $k_{0}, \eta_{0}$ and that $\sum_{s \in S} \delta^{t(s)} u\left(\hat{c}_{s}\right) \pi_{s}<\infty$. Then the following three conditions are equivalent

(1) $\hat{\lambda}_{s}, \hat{k}_{s}, \hat{c}_{s}$ solve the social planner problem for initial condition $k_{0}, \eta_{0}$ 
(2) there exist prices $\hat{q}, \hat{p}$ that satisfy the non-negative profit condition, yielding zero profits for all activities for which $\hat{\lambda}_{s}(a)>0$ and support the consumption plan $\hat{c}_{s}$ with $\sum_{s \in S} \hat{p}_{s} \hat{c}_{s}<\infty$

(3) there exist prices $\hat{q}, \hat{p}$ that satisfy the non-negative profit condition, yielding zero profits for all activities for which $\hat{\lambda}_{s}(a)>0$, the first order conditions and the transversality condition.

Proof: First we observe that if the profit conditions hold, then the transversality condition is equivalent to the consumption plan having finite value. Indeed, from the zero profit condition

$$
q_{0} \hat{k}_{0}-\sum_{s \mid t(s)=T+1} q_{s} \hat{k}_{s}=\sum_{t=0}^{T} \sum_{s \mid t(s)=t+1}\left[q_{s-1} \hat{k}_{s-1}-q_{s} \hat{k}_{s}\right]=\sum_{t=0}^{T} \sum_{s \mid t(s)=t+1} p_{s} \hat{c}_{s} .
$$

Next we consider the $T$-truncated utility economy with utility function

$$
\sum_{s \mid t(s) \leq T} \delta^{t(s)} u\left(c_{s}\right) \pi_{s}+\sum_{s \mid t(s)=T+1} q_{s} k_{s}
$$

We observe that when we eliminate states with zero probability and non-viable kinds of capital, this is an ordinary finite economy. By standard methods, a production plan $\hat{\lambda}_{s}, \hat{k}_{s}, \hat{c}_{s}$ solves the social planner problem if and only if its truncations solve this truncated problem. The equivalence of (2) and (3) to zero now follow immediately from the corresponding facts for the finite horizon case.

\section{Analysis of the Model}

Let us begin by defining the most advanced kind of capital in state $\eta$ to be capital of type $\bar{\omega}(\eta)$ and generation $\bar{i}(\bar{\omega}(\eta), \eta)$ if $m(\eta)>0$, and capital of type $\bar{\omega}(\eta)-1$ and generation $\bar{i}(\bar{\omega}(\eta)-1, \eta)$ if $m(\eta)=0$. Fix the initial condition $\eta_{0}$. We will now analyze the model under three assumptions: first, that the parameters $\rho, \gamma, \delta$ satisfy the hypothesis of Proposition 3.1 so that an optimum exists; second, that $M$ is relatively large compared to $L$; and third, that the initial stock of capital consists solely of the most advanced kind of capital in state $\eta_{0}$. 


\subsection{Basic Results}

It will be helpful to begin with a very simple problem: suppose that capital of a particular type is stalled. More specifically, suppose that there is a single unit of generation 0 capital of this particular type, so that we are contemplating producing some output of the characteristic $t>1$ periods from now on using one of two methods. First, we can simply build more of the existing type and generation of capital until period $t-1$, yielding $\beta^{t-1}$ units of characteristic. Second, we can build a unit of the next type of capital of generation $-L$, then use that to build generation $-L+1$ and so forth until period $t-1$. This yields $\rho^{t-2} \gamma^{t-2-L}$ units of characteristic. If we define

$$
m^{*}=1+\frac{(L+1) \log \gamma+\log \rho}{\log \rho+\log \gamma-\log \beta}
$$

and make the generic assumption that this is not an integer, then for $t<m^{*}$ we would prefer to use the old kind of capital, while for $t>m^{*}$ we would like to use the new kind of capital.

Proposition 4.1: If $M>m^{*}-1$ and the initial stock of capital consists solely of the most advanced kind of capital in state $\eta_{0}$, then with probability 1 in every subsequent state $\eta$ there will be a positive amount of the most advanced kind of capital available. Moreover, there will be a positive amount of at most one other kind of capital, and this will be of the type immediately preceding the most advanced kind. Moreover, the negative state $m(\eta)=0$ can occur only when there is a single type of capital.

Remark: As we shall see below, the equilibrium switches between using a single type of capital to produce consumption and new capital. Then, when that kind of capital is played out, the older kind of capital is used to produce the consumption good and reproduce itself, while the new kind of capital is used solely to produce new generations of itself. Finally, when sufficiently advanced new generations of the new kind of capital are produced, the old kind of capital is abandoned completely, and the cycle begins anew. Of particular interest is the transition when two kinds of capital are in use. We explicitly solve for prices, output and so forth below. However, we should give the intuitive idea of what happens during the transition. 
During the transition, the new kind of capital is relatively unproductive at producing current consumption. However, it is quite productive at producing (indirectly) consumption a number of periods into the future. At equilibrium prices, firms are indifferent between using activities involving either producing consumption from the old kind of capital or activities producing new generations of the new kind of capital. The price at which those new generations can be sold reflects the fact that ultimately they will be used to produce consumption goods. If we think of the new generation of capital as being retained within the firm rather than sold in the market, the firm producing the new generations of capital will have negative cash flow. Since the new kind of capital is not being used to produce consumption, the activity of producing new generations can be viewed as development-learning to produce more advanced generations of capital. The output from this D part of R\&D is more advanced capital, which is either sold or retained within the firm. The activities of Internet startup firms, which have high capitalized value, yet have negative cash flow, can be interpreted as engaging in this development activity: IPOs correspond to selling the relatively advanced generation of capital that has been produced over a number of years within the firm.

Proof: In the good state if there is one kind of capital, then, since $\rho \gamma>\beta$, the next generation should be the only kind produced. On the other hand if the bad state occurs at time $\tau$, then there is no uncertainty about the technology available through period $\tau+M$. Observe that if we produce consumption for period $\tau+t$ by producing the old kind of capital until period $\tau \leq \tau+T<\tau+t-1$ then output of consumption at $\tau+t$ is proportional to $\beta^{T} \rho^{t-2-T} \gamma^{t-2-T-L}$, which, since $\rho \gamma>\beta$, is strictly decreasing in $T$. This implies that it is always best to produce the new capital right away. Now consider $t$ the least integer greater than $m^{*}$. Since $M>m^{*}-1$ there is no uncertainty about the availability of generation $t-L$ of the new type of technology to produce consumption for period $t$ and all subsequent periods. It follows that no capital of the old kind should be produced beginning in period $t-1$ (or of course any later period). Also since $M>t-1$, it is impossible to produce any capital of a higher kind than the newest kind in period $t-1$. Consequently in period $t+1$ the only type of capital is the most advanced kind in that state, giving the desired result. 
Suppose now that only the most advanced kind of capital is available, and denote the most advanced kind of capital in state $\eta$ by $z(\eta)$. Let $V\left(\eta_{s}, k_{s}^{z\left(\eta_{s}\right)}\right)$ be the lifetime value of future consumption beginning in this state with $k_{s}^{z\left(\eta_{s}\right)}$ units of the most advanced capital. Notice that having $k^{i}$ units of generation $i$ capital will simply result in $\gamma^{i} k^{i}$ times as much consumption in every future time and state as with a single unit of generation 0 capital. If we take $V_{m}$ to be the value of a single unit of generation 0 capital (of any type) when $m(\eta)=m$, we can write

$$
\left(\eta_{s}, k_{s}^{z\left(\eta_{s}\right)}\right)=\left(\gamma^{\bar{i}\left(\bar{\omega}\left(\eta_{s}\right), \eta_{s}\right)} k_{s}^{z\left(\eta_{s}\right)}\right)^{-\theta} V_{m\left(\eta_{s}\right)}
$$

for $m>0$ and for $m=0$

$$
\left(\eta_{s}, k_{s}^{z\left(\eta_{s}\right)}\right)=\left(\gamma^{\bar{i}\left(\bar{\omega}\left(\eta_{s}\right)-1, \eta_{s}\right)} k_{s}^{z\left(\eta_{s}\right)}\right)^{-\theta} V_{0}
$$

Notice also for $m>M$ that $V_{m}=V_{M}$.

Notice that capital is produced for period $t$ before the state at period $t$ is known, but is used in period $t$ after the state is determined. Consequently, we can compare the value of the capital stock (relative to the price of consumption) at time $t$ immediately before and immediately after the state is realized. Our goal is to show that good news has a marginal impact on the value of capital, but bad news causes it to change abruptly.

Proposition 4.2: Suppose $M>m^{*}-1$ and the initial stock of capital consists solely of the most advanced kind of capital in state $\eta_{0}$. When $-1 \leq \theta<0$, news of a negative shock $m(\eta)=0$ causes the value of the capital stock to fall immediately. If $\theta>0$ the value of the capital stock rises immediately. Precisely, the ratio of the value of the capital stock after the announcement to that before is given by

$$
\pi+(1-\pi) \frac{V_{0}}{V_{M}} .
$$

Remark: The same method of proof shows that good news causes the stock market to change by 


$$
(1-\pi)+\pi \frac{V_{M}}{V_{0}}
$$

From an economic perspective, the interesting case is when time intervals are relatively short, so that $\pi$ is small. Notice, however, that it is not the case that shorter time intervals lead $V_{0} / V_{M} \rightarrow 1$ : the need to switch to a new technology has an appreciable utility cost regardless of how time is measured. This means that for short time intervals, to a good approximation, good news has little effect on the value of the capital stock, while bad news causes it to change by $V_{0} / V_{M}$.

Proof: Since the capital stock and current consumption are both fixed, the only question is what happens to the price of the capital stock. If in the current period $m(\eta)=0$ it must be that in the previous period $m(\eta)>M$. In addition, by Proposition 4.1 in the current period there is a single type of capital of the most advanced type. Suppose that the amount of this capital is $k_{s}^{z(\eta)}$. If there is not a negative shock in the current period then the value will be

$$
\left(\gamma^{\bar{i}\left(\bar{\omega}\left(\eta_{g}\right), \eta_{g}\right)} k_{s}^{z(\eta)}\right)^{-\theta} V_{M}
$$

if there is a negative shock the value will be

$$
\left(\gamma^{\bar{i}\left(\bar{\omega}\left(\eta_{b}\right)-1, \eta_{b}\right)} k_{s}^{z(\eta)}\right)^{-\theta} V_{0}
$$

The corresponding prices at which capital $k_{s}^{z(\eta)}$ is traded are determined by differentiating these values with respect to $k_{s}^{z(\eta)}$. Notice that $\bar{i}\left(\bar{\omega}\left(\eta_{g}\right), \eta_{g}\right)=\bar{i}\left(\bar{\omega}\left(\eta_{b}\right)-1, \eta_{b}\right)$, since the ability of the capital to produce next period consumption is not changed by the shock. Consequently the price of capital without the negative shock is proportional to $-\theta V_{M}$, and with the negative shock to $-\theta V_{0}$. Clearly $V_{0}<V_{M}$. If $\theta<0$ this means that $-\theta V_{M}>-\theta V_{0}$, while if $\theta>0$ this means that $-\theta V_{M}<-\theta V_{0}$, which is the desired result.

Proposition 4.2 shows that when time intervals are short, good news has a marginal impact on stock prices, while bad news causes them to change abruptly. We reinforce this by showing that once the good state is sufficiently well established, 
meaning $m(\eta) \geq M$, the value of the capital stock from period to period rises exponentially and, in the continuous time limit, continuously.

Proposition 4.3: Suppose $M>m^{*}-1$ and the initial stock of capital consists solely of the most advanced kind of capital in state $\eta_{0}$. When $m\left(\eta_{s}\right) \geq M$

$$
\frac{q_{s}^{z\left(\eta_{s}\right)} k_{s}^{z\left(\eta_{s}\right)} / p_{s}}{q_{s-1}^{z\left(\eta_{s-1}\right)} k_{s-1}^{z\left(\eta_{s-1}\right)} / p_{s-1}}=(\rho \delta)^{\frac{1}{\theta+1}} \gamma^{-\frac{\theta}{\theta+1}}\left((1-\pi)+\pi \frac{V_{0}}{V_{M}}\right)^{\frac{1}{\theta+1}}
$$

Proof: Observe that since $m\left(\eta_{s}\right) \geq M$, the economy is following balanced growth, and in particular the relative price of capital to consumption is not changing. So the growth in the value of capital is due entirely to the increased size of the capital stock:

$$
\frac{q_{s}^{z\left(\eta_{s}\right)} k_{s}^{z\left(\eta_{s}\right)} / p_{s}}{q_{s-1}^{z\left(\eta_{s-1}\right)} k_{s-1}^{z\left(\eta_{s-1}\right)} / p_{s-1}}=\frac{k_{s}^{z\left(\eta_{s}\right)}}{k_{s-1}^{z\left(\eta_{s-1}\right)}} .
$$

Without loss of generality, take $k_{s-1}^{z\left(\eta_{s-1}\right)}=1, \bar{i}\left(\bar{\omega}\left(\eta_{s-1}\right), \eta_{s-1}\right)=0$. Then the Bellman equation is

$$
V_{M}=\delta \max _{\phi} u(\phi)+(\rho \gamma(1-\phi))^{-\theta}\left((1-\pi) V_{M}+\pi V_{0}\right)
$$

The first order condition for the optimum is

$$
\phi^{-\theta-1}+\theta(\rho \gamma)^{-\theta}(1-\phi)^{-\theta-1}\left((1-\pi)+\pi \frac{V_{0}}{V_{M}}\right) V_{M}=0
$$

while from the Bellman equation

$$
V_{M}=-\delta \frac{1}{\theta} \phi^{-\theta}+\delta(\rho \gamma(1-\phi))^{-\theta}\left((1-\pi)+\pi \frac{V_{0}}{V_{M}}\right) V_{M}
$$

or

$$
V_{M}=-\frac{\delta \frac{1}{\theta} \phi^{-\theta}}{1-\delta(\rho \gamma(1-\phi))^{-\theta}\left((1-\pi)+\pi \frac{V_{0}}{V_{M}}\right)}
$$

Substituting back into the first order condition, we find 


$$
\rho(1-\phi)=(\rho \delta)^{\frac{1}{\theta+1}} \gamma^{-\frac{\theta}{\theta+1}}\left((1-\pi)+\pi \frac{V_{0}}{V_{M}}\right)^{\frac{1}{\theta+1}}
$$

which is the desired result.

\subsection{Dynamic Analysis}

We now examine in greater detail the time path of consumption and capital following a negative shock. Without loss of generality we may begin at time 0 in state $\eta_{0}$, assuming that a negative shock has just occurred. We may also assume that there is only one kind capital, that this is type 0 generation 0 , and that there is 1 unit of this capital, as well as $c_{0}$ units of characteristic produced from last period. We also assume that current capital is the most advanced possible given the technology, so the initial state has $\bar{\omega}\left(\eta_{0}\right)=1, i\left(0, \eta_{0}\right)=0, i\left(1, \eta_{0}\right)=-L-1$ and $m\left(\eta_{0}\right)=0$.

In this analysis it is convenient to take $M$ to be the largest integer smaller than $m^{*}$. This means that as soon as the economy switches from two kinds of capital to one, it is possible to have negative shocks once again. This simplifies computations without detracting a great deal from the economics.

Suppose that $0<t \leq M$. Notice that during this period, only the old kind of capital is used to produce consumption, and so from the zero profit condition the market discount factor from period $t$ to period $t+1$ is $p_{t+1} / p_{t}=\beta^{-1}$. In the interior the household must be on the margin between consuming in periods $t$ and $t+1$, so by the first order condition

$$
\delta\left(\frac{c_{t}}{c_{t+1}}\right)^{\theta+1}=\beta^{-1}
$$

From this we can derive the growth rate of consumption from periods 1 to $M$.

$$
\frac{c_{t+1}}{c_{t}}=(\delta \beta)^{\frac{1}{\theta+1}}
$$

During this period the new type of capital will grow at the rate $\rho$, as it is not used to produce consumption. We summarize this information in the table below. 


\begin{tabular}{|l|l|l|l|}
\hline$t$ & $c_{t}$ & $k_{t}^{00}$ & $k_{t}^{1(t-1-L)}$ \\
\hline \hline 0 & $c_{0}$ & 1 & 0 \\
\hline 1 & $c_{1}$ & $k_{1}^{00}$ & $k_{1}^{1(-L)}$ \\
\hline 2 & $(\beta \delta)^{\frac{1}{\theta+1}} c_{1}$ & $k_{2}^{00}$ & $\rho k_{1}^{1(-L)}$ \\
\hline$\vdots$ & $\vdots$ & $\vdots$ & $\vdots$ \\
\hline$M$ & $(\beta \delta)^{\frac{M-1}{\theta+1}} c_{1}$ & $k_{M}^{00}$ & $\rho^{M-1} k_{1}^{1(M-1-L)}$ \\
\hline$M+1$ & $(\beta \delta)^{\frac{M}{\theta+1}} c_{1}$ & 0 & $\rho^{M} k_{1}^{1(M-L)}$ \\
\hline
\end{tabular}

We can also work out the amount of old capital required for $0<t \leq M$ :

$$
k_{t}^{00}=c_{t+1}+\beta^{-1} k_{t+1}^{00} .
$$

From this we can find

$$
k_{1}^{00}=c_{1} \sum_{\tau=0}^{M-1} \beta^{-(M-1-\tau)}(\beta \delta)^{\frac{M-\tau}{\theta+1}} .
$$

We also require that period 1 output be produced from period 0 capital

$$
k_{1}^{1(-L)}=1-c_{1}\left(1+\beta^{-M}(\beta \delta)^{\frac{M}{\theta+1}}\left(\frac{1-\left(\beta(\beta \delta)^{\frac{-1}{\theta+1}}\right)^{M}}{1-\left(\beta(\beta \delta)^{\frac{-1}{\theta+1}}\right)}\right)\right) \text {. }
$$

Finally, observe that initial capital can be used either to produce period 1 consumption, yielding a marginal lifetime utility of $\delta u^{\prime}\left(c_{1}\right)$, or to produce period $M+1$ type 1 capital of generation $M-L$. This yields an equal marginal lifetime utility of

$$
\delta^{M+1} \frac{d\left(\gamma^{M-L} \rho^{M} k_{1}^{1(-L)}\right)^{-\theta} V_{M}}{d k_{1}^{1(-L)}}=\delta u^{\prime}\left(c_{1}\right)
$$


or

$$
-\delta^{M} \theta\left(\gamma^{M-L} \rho^{M}\right)^{-\theta}\left(k_{1}^{1(-L)}\right)^{-\theta-1} V_{M}=c_{1}^{-\theta-1} .
$$

Next we must calculate lifetime utility beginning in the negative state:

$$
V_{0}=-\theta^{-1}\left(c_{1}\right)^{-\theta} \delta \frac{1-\left[\delta(\beta \delta)^{-\frac{\theta}{\theta+1}}\right]^{M+1}}{1-\delta(\beta \delta)^{-\frac{\theta}{\theta+1}}}+\delta^{M+1}\left(\gamma^{M-L} \rho^{M} k_{1}^{1(-L)}\right)^{-\theta} V_{M}
$$

Notice also that in the good state, the fraction of the capital stock devoted to consumption $\phi$ must be constant. Consequently one unit of capital in the good state will produce $\phi \gamma^{i}$ units of the characteristic and $(1-\phi) \rho$ units of the next generation of capital. This leads to the Bellman equation

$$
V_{M}=\delta \max _{\phi} u(\phi)+(1-\pi)(\gamma \rho(1-\phi))^{-\theta} V_{M}+\pi(\rho(1-\phi))^{-\theta} V_{0} .
$$

The first order condition determining $\phi$ is

$$
\phi^{-\theta-1}+\theta \gamma \rho(1-\pi)(\gamma \rho(1-\phi))^{-\theta-1} V_{M}+\theta \rho \pi(\rho(1-\phi))^{-\theta-1} V_{0}=0
$$

or

$$
\phi=\frac{1}{1-\left(\theta(1-\pi)(\gamma \rho)^{-\theta} V_{M}+\theta \pi \rho^{-\theta} V_{0}\right)^{\frac{1}{\theta+1}}},
$$

while the Bellman equation for this value of $\phi$ gives

$$
V_{M}=-\delta \theta^{-1} \phi^{-\theta}+\delta(1-\phi)^{-\theta}(1-\pi)(\gamma \rho)^{-\theta} V_{M}+\delta(1-\phi)^{-\theta} \pi \rho^{-\theta} V_{0}
$$

Solving this for $V_{0}$ we find

$$
V_{0}=\frac{\left(1-\delta(1-\phi)^{-\theta}(1-\pi)(\gamma \rho)^{-\theta}\right) V_{M}+\delta \theta^{-1} \phi^{-\theta}}{\delta(1-\phi)^{-\theta} \pi \rho^{-\theta}} .
$$

The 5 numbered equations must be solved for the unknowns $c_{1}, k_{1}^{1(-L)}, \phi, V_{M}, V_{0}$. By directly substituting (1) into (2) and (3), (3) into (4) and (5), and (4) into (5) this may be reduced to two equations in the unknowns $c_{1}, V_{M}$. 


\subsection{Quantitative Aspects of the Theory}

To get a handle on the implications of the theory, it is useful to consider the extreme case in which bad shocks are rare, so that $\pi \rightarrow 0$, and in which new technologies are difficult to get on line, so that $L \rightarrow \infty$. This will result (for given values of the other parameters) in the most extreme stock market fall.

Using (4) and (5) above, we may explicitly solve for $\phi$ and $V_{M}$ when $\pi=0$ :

$$
\begin{gathered}
1-\phi=\left(\delta(\gamma \rho)^{-\theta}\right)^{\frac{1}{1+\theta}} \\
V_{M}=-\theta^{-1} \frac{\delta}{\left(1-\left(\delta(\gamma \rho)^{-\theta}\right)^{\frac{1}{1+\theta}}\right)^{\theta+1}} .
\end{gathered}
$$

Let $g$ denote the growth rate of consumption (in the good state); then $g=\gamma \rho(1-\phi)$, and we may substitute in the expression for $1-\phi$ to find $\gamma \rho=g^{\theta+1} / \delta$. This gives the value of the capital stock in terms of the growth rate of consumption and the subjective discount factor

$$
V_{M}=-\theta^{-1} \frac{\delta}{\left(1-\left(\delta^{\frac{1-\theta}{1+\theta}} g^{-\theta}\right)\right)^{\theta+1}} .
$$

For the case where $L$ is large, once the technology plays out, the time it takes for a new kind of capital to come on line is excessively long. So we can regard the bad state as similar to the good state, but with a potential growth rate of consumption of $\beta$ rather than $\gamma \rho$. If we let $g_{b}$ denote the essentially fixed growth rate of consumption in this state, we can find $V_{0}$ by replacing $g$ with $g_{b}$ in $V_{M}$. This yields

$$
\frac{V_{0}}{V_{M}}=\frac{\left(1-\left(\delta^{\frac{1-\theta}{1+\theta}} g^{-\theta}\right)\right)^{\theta+1}}{\left(1-\left(\delta^{\frac{1-\theta}{1+\theta}} g_{b}^{-\theta}\right)\right)^{\theta+1}}
$$

Notice that as the logarithmic case is approached, so $\theta \rightarrow 0$, that $V_{0} / V_{M} \rightarrow 1$ as asserted by Proposition 4.1. Notice, however, that it is also the case that as $\theta \rightarrow-1$ (so 
the utility function approaches linearity) $V_{0} / V_{M} \rightarrow 1$. So it is the intermediate range between the logarithm and linear utility functions that is of interest.

Notice that the market rate of interest is $\delta / g^{\theta+1}$ in the good state, and similarly it is $\delta / g_{b}^{\theta+1}$ in the bad state. To explore more carefully, let us suppose that $g_{b}=1$ so that there is no growth following a negative shock. In this case the market rate of interest in the bad state is simply the subjective discount factor. First take this to be $5 \%$, so that $\delta=0.95$. If the rate of growth of consumption in the good state is $5 \%$, we may calculate the variations in the market value of the stock of capital caused by the bad shock for different levels of risk aversion. This is reported in the next table, along with the interest rate in the good and bad states.

\begin{tabular}{|l|l|l|l|l|}
\hline$\theta$ & -.1 & -.2 & -.4 & -.8 \\
\hline $1-V_{0} / V_{M}$ & $6.8 \%$ & $9.9 \%$ & $9.6 \%$ & $1.4 \%$ \\
\hline Interest at $M$ & $9.1 \%$ & $8.6 \%$ & $7.7 \%$ & $5.9 \%$ \\
\hline Interest at 0 & $5.0 \%$ & $5.0 \%$ & $5.0 \%$ & $5.0 \%$ \\
\hline
\end{tabular}

Notice that the size of the market drop in the intermediate range of $\theta$ is not terribly sensitive to the value of $\theta$. In this range the market drops roughly $10 \%$ in response to bad news. If we consider a more drastic fall in the rate of consumption growth from $10 \%$ to $0 \%$, we find the following.

\begin{tabular}{|l|l|l|l|l|}
\hline$\theta$ & -.1 & -.2 & -.4 & -.8 \\
\hline $1-V_{0} / V_{M}$ & $13.4 \%$ & $19.8 \%$ & $19.7 \%$ & $2.9 \%$ \\
\hline Interest at $M$ & $12.8 \%$ & $12.0 \%$ & $10.3 \%$ & $6.8 \%$ \\
\hline Interest at 0 & $5.0 \%$ & $5.0 \%$ & $5.0 \%$ & $5.0 \%$ \\
\hline
\end{tabular}

Here, the market drops on the order of $20 \%$. 
However, in these examples, the market interest rate in the bad state is $5 \%$, which is high by historical standards. If we reanalyze a change from $5 \%$ to $0 \%$ growth with $\delta=.98$, we find the following.

\begin{tabular}{|l|l|l|l|l|}
\hline$\theta$ & -.1 & -.2 & -.4 & -.8 \\
\hline $1-V_{0} / V_{M}$ & $17.8 \%$ & $26.4 \%$ & $27.8 \%$ & $2.9 \%$ \\
\hline Interest at $M$ & $6.2 \%$ & $5.8 \%$ & $4.8 \%$ & $4.4 \%$ \\
\hline Interest at 0 & $2.0 \%$ & $2.0 \%$ & $2.0 \%$ & $3.0 \%$ \\
\hline
\end{tabular}

This means a nearly $30 \%$ drop in the market. The reason that greater patience leads to a more significant market fall is that the bad shock has no consequence for next period consumption, but the difference in future production possibilities grows over time.

While we cannot pretend that the present, simplified model provides a realistic account of observed movements in stock market prices, it is still worthwhile to assess the extent to which the model is roughly consistent with the historical evidence. To this end, we first consider the quality of our simple approximation and then present the results of a simulation of the model for parameter values that are not altogether impossible.

We have not tried to calibrate our model to available U.S. data, as our framework does not incorporate an explicit labor supply rule, nor does it contain other sources of shocks beyond the "large" and infrequent shocks provoked by major technological changes. During the majority of periods in which the existing technology is being improved, there are no other shocks in our model and both quantities and prices follow a deterministic path. Real economies are affected by many other smaller but more frequent shocks that lead to large fluctuations in hours worked, quantities produced and market prices.

Further, we are using a very aggregate model in which only two types of capital are in use at each moment of time. It is hard to imagine that we can approximate real market fluctuations terribly well with a model in which there is a single sector of production and a single technology which is occasionally dismissed to be replaced with a 
new one. A more realistic model would require several capital sectors, each with its own technology shocks, and a more elaborate technology for the production of the final consumption good involving various kinds of capital as well as labor. This would, of course, require an explicit analysis of the flow of resources between sectors in response to shocks, as well as an explicit evaluation of the degree of substitutability among inputs in the various sectors. This is a very interesting task which, nevertheless, goes beyond the scope of the present, theoretical, paper and is left to a future investigation.

To check the quality of the approximation consider the case in which $\delta=0.95$, $\theta=-.4$ and a rate of growth of consumption in the good state of $5 \%$. For comparison, we consider $\pi=0.05, M=12, L=20, \beta=1.052, \rho=1.05$, and $\gamma=1.032$. The growth rate in the bad state is ill defined, so we take $g_{b}=(\delta \beta)^{1 /(\theta+1)}$, which is correct asymptotically. Both sets of results are shown in the table below.

\begin{tabular}{|l|l|l|}
\hline$\theta$ & approximation & exact \\
\hline $1-V_{0} / V_{M}$ & $9.6 \%$ & $9.0 \%$ \\
\hline$g$ & $5.0 \%$ & $5.0 \%$ \\
\hline$g_{b}$ & $0.0 \%$ & $0.0 \%$ \\
\hline
\end{tabular}

Notice the relatively small reduction in the size of the stock market drop, due to the fewer number of periods required to get back to the level of efficiency of the previous technology. (Here $L=20$, as opposed to $L=\infty$ in the approximation.) Similar results can be obtained with other sets of more or less "credible" parameter values. In particular, we have looked at various configurations of parameter values that are consistent with the choice of a quarter as the unit of time. In this case, for discount factors in the range [.98,.99] and choices of $\beta, \gamma, \rho$ and $M$ yielding growth rates of consumption $g \in[1.01,1.03]$ and $g_{b} \in[1.0,1.02]$, we obtain drops in the stock market index that range between 3 and 9 percent over the quarter after the bad news is received.

Finally we have simulated the time paths of aggregate quantities generated by our model over extended periods of time to get an idea of their business cycle properties. The 
figure below reports the time paths of the stock of capital and the flow of consumption generated by a simulation calibrated using the parameters from the last table. Over the time period considered we observe the introduction and consecutive replacement of three different types of capital stocks. The overall pattern is clear and qualitatively acceptable. Accumulation occurs slowly, while dismissal is fast and, in the initial period, abrupt. Observe that when the bad news arrives the process of accumulation stops for a while. During this period only replacement of machines takes place: the old type of capital is used only to produce consumption, while the new kind of machines are accumulated and the sum of the two stocks remains constant from one period to the next. At the end of the replacement period the stock of new machines is equal to the stock of old machines when the recession began, after which net accumulation of capital resumes again. The path of consumption follows along. As predicted, consumption recessions start at the time at which the old technology cannot be improved any further and continue after that for a relatively long number of periods, while the new capital good is improved and accumulated. 
Capital Stock

dotted line is consumption

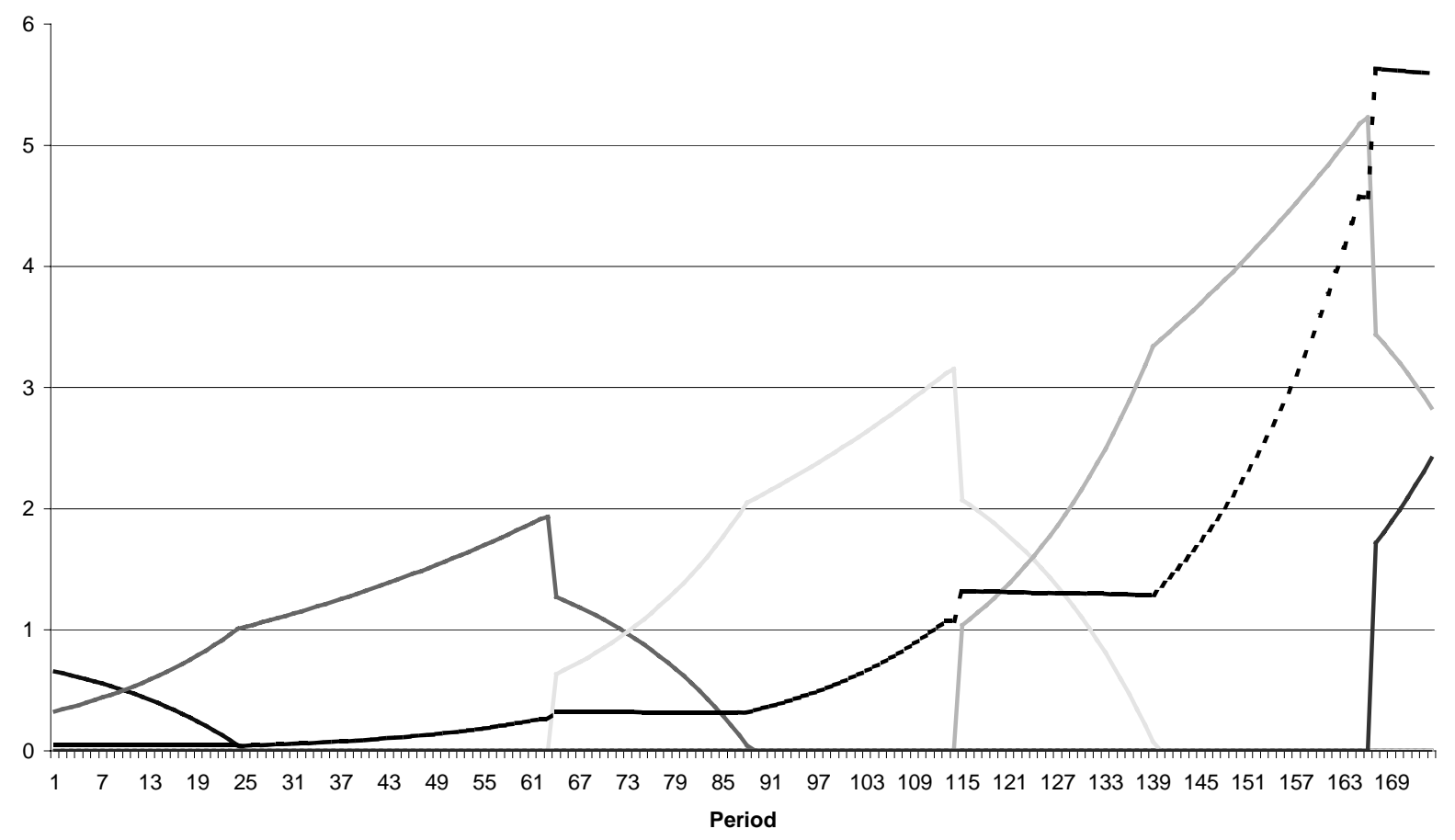

One can distinguish the consumption recession periods, in which there is no growth, and the good periods of high growth beginning when the replacement of the old technology is completed. The cyclical growth pattern of aggregate output can easily be inferred from the separate behavior of $c_{t}$ and $k_{t}$ by recalling that, in the practice of National Income Accounting, output is computed by normalizing relative prices in a base year. In our case, we can, for example, use the relative price of capital at the beginning of the recession period to compute net aggregate output as the sum of the consumption flow plus the net investment flow. The latter is zero during the transition; therefore, output growth would be also nil during this period. When recovery begins, output of the investment good becomes positive and consumption growth resumes; hence, aggregate output growth would increase sharply and converge toward its balanced growth level until the next recession strikes. If, contrary to National Income Accounting practices, one takes into account the changes in the relative prices of capital goods, output growth will be slightly positive even during the recession periods, but still much lower than during the periods of high growth, and persistent oscillations in growth rates will still appear. 
Stock market crashes occur at the beginning of recessions and continue for a while after. Notice that, because of the change in the relative price of capital we just mentioned, stock market valuations begin to recover before consumption growth does. Hence stock market values are good predictors of subsequent movements in total output growth.

\section{Conclusions}

We have constructed a model of long-run growth due to major technological innovations, improvement of existing machines and substitution away from old technologies toward new ones when the former cannot be improved any further. Our theoretical model predicts, in a stylized fashion, that the following sequence of events should be observed.

When an old mode of production (technology) cannot be improved further, it is dismissed and progressively replaced by a newer one, which allows for additional periods of productivity gains and balanced growth. The dismissal of the old technology and the introduction of new machines trigger recessions. They continue for a few periods while the new technology is being developed, but end before development of the new machines is completed. Recoveries settle in slowly and correspond to the phase in which the new capital good is being adopted and improved to its maximum productivity potential. After the initial period, recoveries accelerate, leading to a phase of balanced growth that lasts until the next innovation takes place. Hence, recessions are shorter than recoveries, but more abrupt. The growth rates of aggregate output persistently oscillate around a kind of average balanced growth rate. As in other models of growth and innovation, such as that of Aghion and Howitt [1], recessions are a consequence of the adoption of new technologies. Contrary to those models, recessions are not due to the fact that labor invested in $\mathrm{R} \& \mathrm{D}$ does not contribute to aggregate output. They are instead due to the sudden drop in the ouptut of the investment good and to the reduction in the growth rate of the consumption sector. We find this characterization more in line with what is currently known about business cycles and growth.

The behavior of stock market prices predicted by our model is rather interesting and seems to correspond, qualitatively and quantitatively, to patterns that can be observed in long-run data. Sizeable stock market crashes occur from time to time: they are larger in 
magnitude than booms, but less frequent and last for fewer periods. The stock market index grows somewhat smoothly for many periods and then collapses for a few periods. Our quantitative exercise shows that, for reasonable parameter values, the size of the stock market crashes may be as high as $30 \%$ and as low as 4 or $5 \%$. These large and sudden crashes, which follow periods of regular growth, are due to fundamental economic factors and not to market inefficiencies or investors' irrational behavior. 


\section{References}

1. Aghion, P. and P. Howitt, "On the Microeconomic Effects of Technological Change," University College London, (1996).

2. Atkeson, A. and P. Kehoe, "Evolution and Transition: the Role of Informational Capital," Federal Reserve Bank of Minneapolis Staff Report \#162, (1993).

3. Boldrin, M., L. Christiano and J. Fisher, "Habit Persistence and Asset Returns in an Exchange Economy," Macroeconomic Dynamics, 1, (1997), 312-332.

4. Boldrin, M. and D. K. Levine, "Growth Under Perfect Competition," mimeo, Universidad Carlos III and UCLA, (November 1997).

5. Crafts, N., "The Industrial Revolution," in The Economic History of Britain Since 1700, (R. Flood and D. McCloskey, Eds.), Cambridge University Press, New York, (1994).

6. David, P., "The Dynamo and the Computer: An Historical Perspective on the Productivity Paradox," American Economic Review, 80, (1990), 355-361.

7. Gali, J., "Technology, Employment and the Business Cycle," American Economic Review, 89, (1999), 249-271.

8. Greenwood, J. and B. Jovanovic, "The Information Technology Revolution and the Stock Market: Preliminary Evidence from the CRSP Data," University of Rochester, 1999.

9. Greenwood, J. and B. Jovanovic, "The IT Revolution and the Stock Market," University of Rochester, (1999).

10. Greenwood, J. and A. Yorukoglu, “1974,” University of Rochester, (1996).

11. Hornstein, A. and P. Krusell, "Can Technology Improvements Cause Productivity Slowdowns?" mimeo, University of Rochester, (1996).

12. Jovanovic, B. and R. Rob, "Long Waves and Short Waves," Econometrica, 58, (1990), 1391-1409. 
13. Lee, I. H., "Market Crashes and Informational Avalanches," Review of Economic Studies, 65, (1998), 741-760.

14. Lucas, R. E., Jr., “Asset prices in an exchange economy,” Econometrica, 46, (1978) 1431-1445.

15. Mansfield, E., Industrial Research and Technological Innovation, Norton: New York, 1968.

16. Mehra, R. and E. Prescott, "The Equity Premium: a Puzzle," Journal of Monetary Economics, 15, (1985), 145-161.

17. Shiller, R., Market Volatility, MIT Press: Cambridge, MA, 1989.

18. Zeira, J., "Informational Overshooting, Booms and Crashes," Journal of Monetary Economics, 43, (1999), 237-258 . 


\section{Appendix: Stock Market Data}

For the years 1889-1984, the table below reports the S\&P 500 index divided by GNP deflator, and the deviation from the mean of the difference between the log of this value and that of the subsequent year. The data are from Shiller [17].

\begin{tabular}{|r|r|r|}
\hline Year & S\&P & Growth \\
\hline 1889 & 0.27 & $3.0 \%$ \\
\hline 1890 & 0.28 & $-8.6 \%$ \\
\hline 1891 & 0.26 & $14.0 \%$ \\
\hline 1892 & 0.30 & $0.1 \%$ \\
\hline 1893 & 0.31 & $-21.3 \%$ \\
\hline 1894 & 0.25 & $-0.8 \%$ \\
\hline 1895 & 0.25 & $3.6 \%$ \\
\hline 1896 & 0.26 & $-3.4 \%$ \\
\hline 1897 & 0.26 & $11.8 \%$ \\
\hline 1898 & 0.29 & $19.0 \%$ \\
\hline 1899 & 0.36 & $-5.4 \%$ \\
\hline 1900 & 0.34 & $15.3 \%$ \\
\hline 1901 & 0.40 & $8.1 \%$ \\
\hline 1902 & 0.44 & $3.5 \%$ \\
\hline 1903 & 0.46 & $-27.1 \%$ \\
\hline 1904 & 0.36 & $20.7 \%$ \\
\hline 1905 & 0.44 & $13.9 \%$ \\
\hline & & \\
\hline
\end{tabular}

\begin{tabular}{|r|r|r|}
\hline 1906 & 0.51 & $-8.8 \%$ \\
\hline 1907 & 0.47 & $-34.6 \%$ \\
\hline 1908 & 0.34 & $23.4 \%$ \\
\hline 1909 & 0.43 & $7.9 \%$ \\
\hline 1910 & 0.47 & $-8.9 \%$ \\
\hline 1911 & 0.44 & $-7.2 \%$ \\
\hline 1912 & 0.41 & $1.5 \%$ \\
\hline 1913 & 0.42 & $-13.2 \%$ \\
\hline 1914 & 0.37 & $-15.6 \%$ \\
\hline 1915 & 0.32 & $9.4 \%$ \\
\hline 1916 & 0.36 & $-20.7 \%$ \\
\hline 1917 & 0.29 & $-42.7 \%$ \\
\hline 1918 & 0.19 & $7.7 \%$ \\
\hline 1919 & 0.21 & $-0.2 \%$ \\
\hline 1920 & 0.21 & $-8.0 \%$ \\
\hline 1921 & 0.20 & $6.0 \%$ \\
\hline 1922 & 0.21 & $16.4 \%$ \\
\hline 1923 & 0.25 & $-0.9 \%$ \\
\hline 1924 & 0.25 & $14.6 \%$ \\
\hline & & \\
\hline
\end{tabular}

\begin{tabular}{|r|r|r|}
\hline 1925 & 0.30 & $15.6 \%$ \\
\hline 1926 & 0.35 & $8.0 \%$ \\
\hline 1927 & 0.38 & $24.9 \%$ \\
\hline 1928 & 0.50 & $34.7 \%$ \\
\hline 1929 & 0.71 & $-12.2 \%$ \\
\hline 1930 & 0.63 & $-21.6 \%$ \\
\hline 1931 & 0.52 & $-55.0 \%$ \\
\hline 1932 & 0.30 & $-12.5 \%$ \\
\hline 1933 & 0.27 & $31.0 \%$ \\
\hline 1934 & 0.37 & $-16.6 \%$ \\
\hline 1935 & 0.32 & $37.6 \%$ \\
\hline 1936 & 0.46 & $20.0 \%$ \\
\hline 1937 & 0.57 & $-43.4 \%$ \\
\hline 1938 & 0.37 & $9.8 \%$ \\
\hline 1939 & 0.42 & $-3.7 \%$ \\
\hline 1940 & 0.41 & $-23.1 \%$ \\
\hline 1941 & 0.33 & $-28.9 \%$ \\
\hline 1942 & 0.25 & $2.2 \%$ \\
\hline 1943 & 0.25 & $9.6 \%$ \\
\hline
\end{tabular}




\begin{tabular}{|c|c|c|c|c|c|}
\hline 1944 & 0.28 & $8.4 \%$ & 1968 & 1.10 & $1.5 \%$ \\
\hline 1945 & 0.31 & $21.3 \%$ & 1969 & 1.13 & $-17.6 \%$ \\
\hline 1946 & 0.39 & $-27.9 \%$ & 1970 & 0.96 & $-2.5 \%$ \\
\hline 1947 & 0.30 & $-9.3 \%$ & 1971 & 0.94 & $5.0 \%$ \\
\hline 1948 & 0.27 & $3.2 \%$ & 1972 & 1.00 & $6.2 \%$ \\
\hline 1949 & 0.28 & $7.0 \%$ & 1973 & 1.07 & $-32.0 \%$ \\
\hline 1950 & 0.31 & $12.8 \%$ & 1974 & 0.79 & $-36.8 \%$ \\
\hline 1951 & 0.35 & $10.6 \%$ & 1975 & 0.55 & $22.4 \%$ \\
\hline 1952 & 0.40 & $4.6 \%$ & 1976 & 0.70 & $-0.2 \%$ \\
\hline 1953 & 0.42 & $-5.7 \%$ & 1977 & 0.70 & $-22.0 \%$ \\
\hline 1954 & 0.40 & $31.8 \%$ & 1978 & 0.57 & $0.3 \%$ \\
\hline 1955 & 0.56 & $18.5 \%$ & 1979 & 0.58 & $-0.2 \%$ \\
\hline 1956 & 0.68 & $-1.3 \%$ & 1980 & 0.58 & $8.9 \%$ \\
\hline 1957 & 0.68 & $-13.6 \%$ & 1981 & 0.64 & $-19.0 \%$ \\
\hline 1958 & 0.60 & $27.7 \%$ & 1982 & 0.54 & $15.6 \%$ \\
\hline 1959 & 0.79 & $1.1 \%$ & 1983 & 0.63 & $9.5 \%$ \\
\hline 1960 & 0.81 & $0.6 \%$ & 1984 & 0.70 & \\
\hline 1961 & 0.82 & $12.1 \%$ & & & \\
\hline 1962 & 0.94 & $-8.5 \%$ & & & \\
\hline 1963 & 0.87 & $13.5 \%$ & & & \\
\hline 1964 & 1.00 & $8.7 \%$ & & & \\
\hline 1965 & 1.11 & $3.5 \%$ & & & \\
\hline 1966 & 1.16 & $-13.6 \%$ & & & \\
\hline 1967 & 1.02 & $6.6 \%$ & & & \\
\hline
\end{tabular}

